\title{
L'apport de l'endoscopie naso-sinusienne micro-invasive dans l'extraction des dents ou des racines accidentellement refoulées dans le sinus maxillaire: à propos d'un cas clinique
}

\author{
Boukais $\mathbf{H}^{\mathbf{1}}$, Ouenoughi. $\mathbf{K H}^{\mathbf{2}}$, Zerrouki $\mathbf{W}^{\mathbf{1}}$, Haraoubia $\mathrm{MS}^{\mathbf{2}}$, Zemirli $\mathbf{O}^{\mathbf{2}}$ \\ ${ }^{1}$ Service de Pathologie et Chirurgie buccale, CHU Béni-Méssous, Alger, Algérie \\ ${ }^{2}$ Service d'ORL, CHU Béni-Méssous, Alger, Algérie \\ h.boukais@yahoo.fr
}

Depuis plus de 20 ans, les indications de la chirurgie sinusienne et endonasale vidéoendoscopique n'ont cessé de s'élargir et remplacent les voies d'abord externes et intrabuccales, plus invasives et délabrantes. Les polyposes naso-sinusiennes, les sinusites chroniques bactériennes et fongiques sont des pathologies qui bénéficient aussi de cette technique. D'autres spécialités comme la chirurgie maxillo-faciale et l'odontostomatologie sont deux disciplines qui ont également bénéficié des progrès de cette technique, par son application à des pathologies qui leur sont propres.

Autrefois, l'extirpation des corps étrangers intra-sinusiens comme les dents ou les racines refoulées accidentellement lors de l'extraction, se faisait par le recours à une intervention chirurgicale très invasive et délabrante: La technique de Cadwell Luc entraîne des lésions osseuses (maxillaire) et muqueuses (muqueuse sinusienne), et comporte un risque d'infection du site opératoire à partir des germes commensaux de la cavité buccale (cavité fortement septique).

A travers notre communication affichée, nous illustrerons un cas clinique d'extirpation par la technique d'endoscopie sinusienne, d'une dent de sagesse maxillaire refoulée dans le sinus lors de sa tentative d'extraction. L'intérêt de cette technique est de préserver les propriétés physiologiques de la muqueuse sinusienne permettant de garantir une bonne ventilation nasale et sinusienne. 